\title{
Experimental Non-Local Generation of Entanglement from Independent Sources*
}

\author{
Xian-Min Jin ${ }^{1,2 * *}$, Jügen Röch ${ }^{2}$, Juan Yin ${ }^{1}$, Tao Yang ${ }^{1}$ \\ ${ }^{1}$ Hefei National Laboratory for Physical Sciences at Microscale and Department of Modern Physics, \\ University of Science and Technology of China, Hefei 230026 \\ ${ }^{2}$ Physikalisches Institut, Ruprecht-Karls-Universität Heidelberg, Philosophenweg 12, 69120 Heidelberg, Germany
}

\begin{abstract}
We experimentally demonstrate a non-local generation of entanglement from two independent photonic sources in an ancilla-free process. Two bosons (photons) are entangled in polarization space by steering into a novel interferometer setup, in which they have never meet each other. The entangled photons are delivered to polarization analyzers in different sites, respectively, and a non-local interaction is observed. Entanglement is further verified by the way of the measured violation of a CHSH type Bell's inequality with S-values of 2.54 and 27 standard deviations. Our results will shine a new light into the understanding on how quantum mechanics works, have possible philosophic consequences on the one hand and provide an essential element for quantum information processing on the other hand. Potential applications of our results are briefly discussed.
\end{abstract}

PACS numbers: 03.65.Ud, 03.67.Mn, 42.50.Dv

Entanglement is considered to be one of the most profound features of quantum mechanics and is extremely important in quantum information. It lies at the heart of the Einstein-Podolsky-Rosen paradox, [1] Bell's inequalities, [2] and the nonlocality of quantum mechanics, and has comprehensive application in quantum communication [3, 4] and quantum computation. [5] So far, several methods to generate entanglement have been proposed and realized, as illustrated in Figs. 1(a) and 1(b). One way is to entangle two particles which root in a common source, [6-8] such as from the process of spontaneous parametric down conversion (SPDC) in a nonlinear optical crystal, where a ultraviolet pump photon decays with low probability into two infrared photons in terms of conservation of energy and momentum (Fig. 1(a)). The other way is to have two particles interacting with each other, [9] typically, oppositely polarized photons, say horizontal $(\mathrm{H})$ and vertical (V), impinging on a beamsplitter (BS) from opposite input ports, photons can be entangled by exchange interaction and postselecting photons from opposite output ports, i.e. the well-known Hong-Ou-Mandel (HOM) [10] interference (Fig. 1(b)). An otherguess idea to obtain entanglement has been proposed [11-13] and realized experimentally by Pan et al.,[14] i.e. the so-called entanglement swapping (Fig. 1(c)), which entangles freely propagating particles that never physically interacted with one another or which have never been dynamically coupled by any other means. As is shown in Fig. 1(c), two simultaneously produced entangled photons, pair EP1-EP2 and pair EP3-EP4. One photon from each pair (photon EP2 and EP3) is subjected to a Bell-state measurement (BSM) which can be realized with a BS and a coincidence measurement between two outputs. This results in projecting the other two outgoing photons EP1 and EP4 into an

${ }^{*}$ Supported by the Marie Curie Excellence Grant of the EU, Chinese Academy of Sciences, and the National Basic Research Program under Grant No 2006CB921900.

**Email: jinxm@ustc.edu.cn

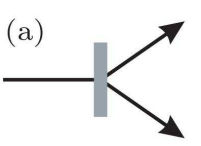

(b)

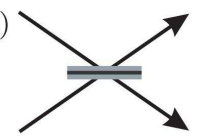

(d)

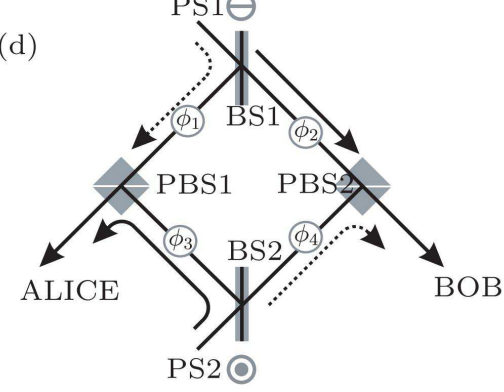

FIG. 1: Method of non-local generation of entanglement. (a) Entanglement rises from the process of spontaneous parametric down conversion by pumping a nonlinear crystal. (b) Entangle two photons by employing HOM interference. (c) Entanglement swapping. (d) Our scheme of non-local generation of entanglement from independent photonic sources. The solid and dotted lines indicates the two cases we select. NLC: nonlinear crystal; BS: beamsplitter; PBS: polarized beamsplitter; EP1,EP2,EP3,EP4: entangled photons 1,2,3,4; PS1,PS2: independent photonic sources 1, 2.

entangled state.

A marvelous scheme proposed by Yurke et al.[15] shows that a kind of Bell-type inequality violation occurs even between two independent particle sources, and an interference between two indistinguishable electrons from independent sources has been realized by Neder et al. [16] In this Letter, we experimentally demonstrate a non-local generation of entanglement from independent photonic sources, which is totally different from the means mentioned above. By using a novel apparatus [15, 17] and post-selection technology we obtain an entangled state from two independent photons without coming from a common source, interacting in the past or ancillary 
entangled states.

A diagram of our scheme is shown in Fig. 1(d), two independent, separated, photonic sources PS1 and PS2 with polarization $\mathrm{H}$ and $\mathrm{V}$ are delivered into input ports of the 50:50 beamsplitters BS1 and BS2, respectively. The other input ports of BS1 and BS2 serve to vacuum. After the action of the BS1 and BS2, the single photons from PS1 and PS2 will be split coherently to two spatial modes of each BS in a superposition style as:

$$
\begin{aligned}
& |\psi\rangle_{1}=|H\rangle_{1} \otimes \frac{1}{\sqrt{2}}\left(i A_{1}^{\dagger}+B_{1}^{\dagger}\right)|0\rangle, \\
& |\psi\rangle_{2}=|V\rangle_{2} \otimes \frac{1}{\sqrt{2}}\left(i A_{2}^{\dagger}+B_{2}^{\dagger}\right)|0\rangle,
\end{aligned}
$$

where we have used the convention to express the output state of BS1 and BS2 in the particle creation operators. The factor $i$ is a consequence of unitarity. It corresponds physically to a phase jump upon reflection at a BS. Equation (1) describes the fact that PS1 (PS2) can be found with equal probability (50\%) in either of the output modes to Alice $A_{1}\left(A_{2}\right)$ or to Bob $B_{1}$ $\left(B_{2}\right)$. The four output beams in mode $A_{1}, B_{1}, A_{2}, B_{2}$ will undergo a corresponding phase shift $\phi_{1}, \phi_{2}, \phi_{3}, \phi_{4}$, then recombine and superpose at polarizing beamsplitter (transmit $\mathrm{H}$ and reflect V) PBS1 and PBS2 with a partnership $\left(A_{1}, A_{2}\right) \Rightarrow \mathrm{A}$ and $\left(B_{1}, B_{2}\right) \Rightarrow \mathrm{B}$. Considering similar unitary transformation for reflection from PBS, the state in Eq. (1) thus evolves into:

$$
\begin{aligned}
& \left|\psi^{\prime}\right\rangle_{1}=|H\rangle_{1} \otimes \frac{1}{\sqrt{2}}\left(i e^{i \phi_{1}} A_{1}^{\dagger}+e^{i \phi_{2}} B_{1}^{\dagger}\right)|0\rangle, \\
& \left|\psi^{\prime}\right\rangle_{2}=|V\rangle_{2} \otimes \frac{1}{\sqrt{2}}\left(-e^{i \phi_{3}} A_{2}^{\dagger}+i e^{i \phi_{4}} B_{2}^{\dagger}\right)|0\rangle .
\end{aligned}
$$

It should be noted that photons PS1 and PS2 are not distinguishable anymore if they arrive at the PBS1 or PBS2 simultaneously. The spatial parts of photons $A_{1}$ and $A_{2}\left(B_{1}\right.$ and $\left.B_{2}\right)$ will be integrate to A (B) with the action of PBS1 (PBS2). The total outgoing state including both the spatial and the spin part will thus be written as

$$
\begin{aligned}
& \left|\psi^{\prime}\right\rangle_{1} \otimes\left|\psi^{\prime}\right\rangle_{2} \\
& =\frac{1}{\sqrt{2}}\left(-i e^{i\left(\phi_{1}+\phi_{3}\right)}|H\rangle_{A}|V\rangle_{A}+i e^{i\left(\phi_{2}+\phi_{4}\right)}|H\rangle_{B}|V\rangle_{B}\right. \\
& \left.-\left(e^{i\left(\phi_{1}+\phi_{4}\right)}|H\rangle_{A}|V\rangle_{B}+e^{i\left(\phi_{2}+\phi_{3}\right)}|V\rangle_{A}|H\rangle_{B}\right)\right) .
\end{aligned}
$$

With the post selection technology, Alice and Bob just pick out the events only one photon in each site. Omitting an overall phase shift, the final two-photon state shared between Alice and Bob will be a tunably polarization-entangled state:

$$
|\Psi\rangle_{A B}=\frac{1}{\sqrt{2}}\left(|H\rangle_{A}|V\rangle_{B}+e^{i \phi}|V\rangle_{A}|H\rangle_{B}\right),
$$

where $\phi=\phi_{2}+\phi_{3}-\phi_{1}-\phi_{4}$. By properly adjusting our apparatus such that $\phi=0$ or $\pi$, the generated state shared by Alice and Bob will be exactly maximal polarization-entangled state.

$$
\begin{aligned}
& \left|\Psi^{+}\right\rangle_{A B}=\frac{1}{\sqrt{2}}\left(|H\rangle_{A}|V\rangle_{B}+|V\rangle_{A}|H\rangle_{B}\right), \\
& \left|\Psi^{-}\right\rangle_{A B}=\frac{1}{\sqrt{2}}\left(|H\rangle_{A}|V\rangle_{B}-|V\rangle_{A}|H\rangle_{B}\right) .
\end{aligned}
$$

In the process, PS1 (PS2) has the same probability $P=0.5$ to pass the BS1 (BS2) or to be reflected. Thus, four different possibilities arise: (1) PS1 $\Rightarrow \mathrm{A}, \mathrm{PS} 2 \Rightarrow \mathrm{A}$; (2) PS1 $\Rightarrow \mathrm{B}$, PS2 $\Rightarrow \mathrm{B}$; (3) $\mathrm{PS} 1 \Rightarrow \mathrm{A}, \mathrm{PS} 2 \Rightarrow \mathrm{B}$; (4) PS1 $\Rightarrow \mathrm{B}, \mathrm{PS} 2 \Rightarrow \mathrm{A}$. Each of the four cases occurs with the same probability. The cases (1) and (2), in which PS1 and PS2 meet with each other, are filtered by our conditional trigger strategy, therefore have no contribution to our finally obtained state in Eq. (5). The cases (3) and (4) are selected by Alice and Bob, whose trajectories are shown in Fig. 1(d), with the dotted line for the case (3) and the solid line for the case (4). In both the cases, PS1 and PS2 always fly to opposite directions so that they never meet each other. However, the cases (3) and (4) themselves are not differentiable and can be indicated quantum mechanically in the superposition of these two cases, i.e. entanglement.

A schematic drawing of the experimental realization and analyzing of the non-locally generated entangled state is shown in Figs. 2(a) and 2(b). Our scheme works ideally with true independent single photon input. In our proof-ofprinciple experiment, we employ disentangled photons from SPDC sources as the two input photons. As shown in Fig. 2(a), an argon-ion UV laser beam (with a power of $300 \mathrm{mw}$, a waist of $80 \mu \mathrm{m}$ and a central wavelength of $351.1 \mathrm{~nm}$ ) passes through a $2 \mathrm{~mm}$ beta-barium-borate (BBO) crystal to generate photonic pairs $(702.2 \mathrm{~nm})$ with type-II phase matching. Unwanted laser-fluorescence is minimized by a dispersion prism. With narrow bandwidth interference filters (NIF) of $1.5 \mathrm{~nm}$ in front of single photon detectors, we collect about 12000 pairs of photons per second, and about 150000 per second for single photon counts in each side. Down-converted extraordinary and ordinary photons have different velocities and travel along different paths inside the crystal due to the birefringent effect of the BBO crystal. The resulting walk-off effects are compensated by a combination of a half wave plate $(\lambda / 2, H W P)$ and an additional $1 \mathrm{~mm}$ BBO crystal in each arm. In this step, by checking the entanglement visibility between the two created photons we can obtain exactly identical photons in frequency with the assistance of type-II phase matching. Further followed by a pair linear polarizers POL1 and POL2 (extinction ratio of 10000:1) setting at $0^{\circ}$ and $90^{\circ}$, two independent photons with $\mathrm{H}$ and $\mathrm{V}$ polarization respectively, which are located at different sites and with accurately the same frequency, will be obtained.

As is shown in Fig. 2(b). The prepared two photons are delivered into the novel interferometer we have illustrated above. At the output ports of the interferometer located in Alice and Bob, the polarization analyzers are employed to measure ingoing photons. We use $1.5 \mathrm{~nm}$ NIFs to increase the coherent length, define the exact spectral mode and remove all background light. Highly extinction-ratio polarizer Pol3 (Pol4) is 


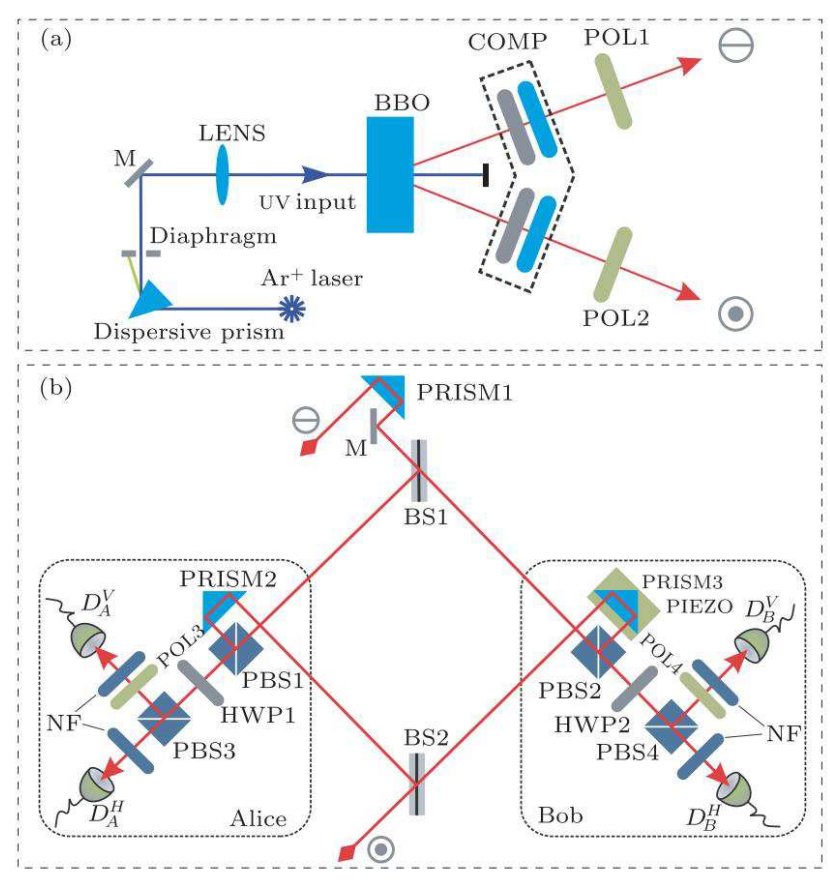

FIG. 2: Schematic of the experimental setup. (a) Preparation of two photonic sources. A UV beam from argon-ion laser $(351.1 \mathrm{~nm}, 300$ $\mathrm{mW}$ ) is directed into the BBO crystal to create photon pairs with wavelength $702.2 \mathrm{~nm}$. Two compensators (COMP) are used to offset the birefringent effect caused by the $\mathrm{BBO}$ crystal during parametric down-conversion. Polarizers POL1 and POL2 setting at $0^{\circ}$ and $90^{\circ}$ to prepare single photon $\mathrm{H}$ and $\mathrm{V}$ in different spatial modes. (b) The setup for nonlocally creating entanglement. The PRISM1,PRISM2 and PRISM3 are built in micrometer-precision manual positioning stages to balance path length for PS1(H) and PS2(V) to PBS1 and PBS2, and an additional nanometer stepsize piezo translation stage is mounted to PRISM2 to tune or fix $\phi$ arbitrarily and therewith has full control over the phase. A polarization analyzer in Alice (Bob) side comprises an HWP, a PBS and two single-photon counting modules (Perkin-Elmer, SPCM-AQR-13 operated in Geiger-mode).)

used to eliminate the imperfect reflection of PBS1 (PBS2) for Vertical polarization.

One prerequisite to observe nonlocal interaction is to ensure that the two input photons are indistinguishable at each side. Perfect spatial and temporal overlaps on the PBS1 and PBS2 are necessary, which are highly related to the visibility of the created entangled states. Experimentally, all the photons are collected with single-mode fibers to define the exact spatial mode. All NIFs are set in front of each detector to define the exact spectral mode. Additional prism 1, prism 2 and prism 3 built in micrometer-precision manual positioning stages are employed to achieve accurate temporal overlap for two photons on PBS1 and PBS2. To check whether the condition of arriving at PBS1 and PBS2 at the same time for two photons is fulfilled, we exploit and apply HOM type interference [10] at the both of Alice and Bob's sites. The first and second terms of Eq. (3) denote the cases that both photons reach the same site. These rejected parts can be reused
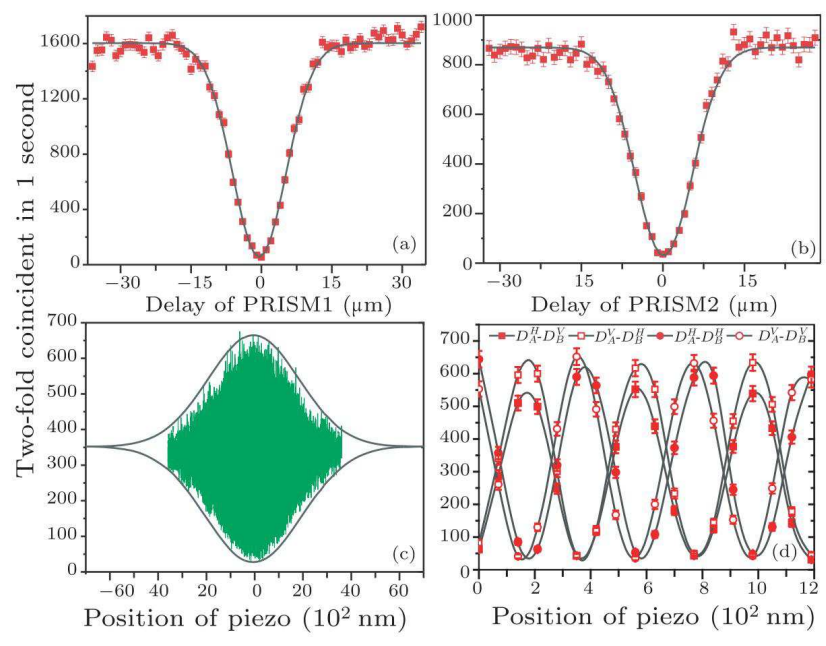

FIG. 3: Demonstration of the way to make two photons indistinguishable and the non-local interference fringe. (a) and (b) HongOu-Mandel dips observed in Alice's side (a) and Bob's side (b). (c) The envelope of the observed two-fold coincidence of $D_{A}^{H}-D_{B}^{V}$. (d) We use a piezo translation stage to move PRISM2 to perform a fine scan around the centre of the envelope. By setting the piezo system to a position where we observe maximum two-fold coincidence of $D_{A}^{H}-D_{B}^{V}$, we can achieve $\phi=\pi$.

to confirm the simultaneous arrival of two photons. Considering the indistinguishability of the two photons, both the terms can be rewritten in $|+\rangle /|-\rangle$ basis as $|+\rangle_{1}|+\rangle_{2}+|-\rangle_{1}|-\rangle_{2}$. The optical axis of HWP1 and HPW2 are rotated to $22.5^{\circ}$, the photon with $|+\rangle$ polarization will fire the detectors $D_{A}^{H}$ and $D_{B}^{H}$, the one with $|-\rangle$ polarization will fire $D_{A}^{V}$ and $D_{B}^{V}$ definitely. Thus a HOM-type dip will emerges at perfect temporal overlap if coincidence measurements of $D_{A}^{H}-D_{A}^{V}\left(D_{B}^{H}-D_{B}^{V}\right)$ are performed. By adjusting prisms 1 and 2, we have measured the two-fold coincident counts as a function of scanning position of prisms 1 and 2. Ideally, there should be completely destructive interference if the wavepackets of the two photons overlap perfectly. However, it is difficult to make the two wavepackets absolutely identical or exactly overlapped in practice. In our experiment, we achieve a very high visibility of the dips $V_{A}=\left(C_{\text {plat }}-C_{\text {dip }}\right) / C_{\text {plat }}=(96.6 \pm 0.5) \%$ for Alice and $V_{B}=(95.9 \pm 0.8) \%$ for Bob respectively, where $C_{\text {plat }}$ is the non-correlated coincidence rate at the plateau and $C_{d i p}$ is the interfering coincidence rate at the dip, see Figs. 3(a) and 3(b). The extremely high HOM-type interference visibility ensure considerably high entanglement visibility we can obtain.

Once the indistinguishability of two photons are fulfilled at both sites, we measure the two-fold coincidence between the output modes toward detectors $D_{A}^{H}$ and $D_{B}^{V}$ with HWP1 and HWP2 setting at $22.5^{\circ}$. An interference fringe can be observed by performing fine adjustment of the position of either PRISM1 or PRISM2. By using a piezo translation stage (minimum step size $1 \mathrm{~nm}$ ) to move prism 2, we perform a fine scan to measure the envelope of the two-fold coincidence $D_{A}^{H}-D_{B}^{V}$ with a step size of $50 \mathrm{~nm}$, see Fig. 3(c). By setting the piezo system to a position where we observe maximal two-fold co- 


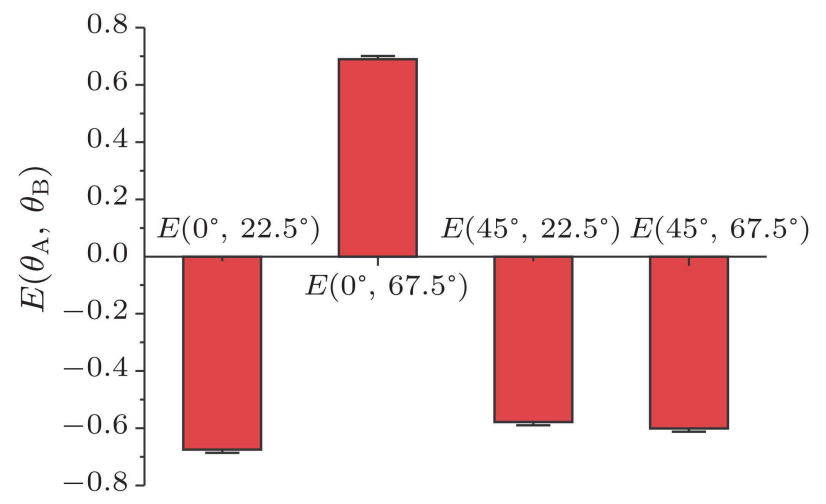

FIG. 4: Experimentally measured correlation function $E$ required for $\mathrm{CHSH}$ inequality. Every measurement of $E$ is finished within $3 \mathrm{~s}$. These data yield the Bell parameter $S=2.54 \pm 0.02$ which is in confliction with local realism by over 27 standard deviations. The error bars denote one standard deviation, deduced from propagated Poissonian counting statistics of the raw detection events.

incidence of $D_{A}^{H}-D_{B}^{V}$ as shown in Fig. 3(d), we can achieve $\phi=\pi$ and obtain the $\left|\Psi^{-}\right\rangle_{A B}$ state.

The two-photon entangled state shared by Alice and Bob can be verified by a Clauser-Horne-Shimony-Holt (CHSH) type inequality, [18] which is one type of the Bell inequalities. The polarization correlation function is defined as follows:

$$
E\left(\theta_{A}, \theta_{B}\right)=\frac{N_{++}+N_{--}-N_{+-}-N_{-+}}{N_{++}+N_{--}+N_{+-}+N_{-+}},
$$

where $N_{++}, N_{--}, N_{+-}$and $N_{-+}$are the coincident counts between Alice and Bob with the actual settings of $\left(\theta_{A}, \theta_{B}\right)$, $\left(\theta_{A}+\pi / 2, \theta_{B}+\pi / 2\right),\left(\theta_{A}, \theta_{B}+\pi / 2\right)$ and $\left(\theta_{A}+\pi / 2, \theta_{B}\right)$, respectively. In the CHSH inequality, parameter $S$ is defined as

$$
S=\left|E\left(\theta_{A}, \theta_{B}\right)-E\left(\theta_{A}, \theta_{B}^{\prime}\right)+E\left(\theta_{A}^{\prime}, \theta_{B}\right)+E\left(\theta_{A}^{\prime}, \theta_{B}^{\prime}\right)\right| .
$$

In the local realistic view, no matter what angles $\theta_{A}$ and $\theta_{B}$ are set to, parameter $S$ should be $S \leqslant 2$. However, in the view of quantum mechanics, $S$ will reach the maximal value $2 \sqrt{2} \approx 2.828$ when the polarization angles are set to $\left(\theta_{A}, \theta_{A}^{\prime}, \theta_{B}, \theta_{B}^{\prime}\right)=\left(0^{\circ}, 45^{\circ}, 22.5^{\circ}, 67.5^{\circ}\right)$. The observed values of the correlation functions are shown in Fig.4, resulting in $S=2.54 \pm 0.02$, which violates Bell's inequality by 27 standard deviations. This clearly confirms the quantum nature of the entanglement state.

Ultra high stability has been a major challenge for our experiment. The interferometer's geometry has carefully been designed in a way which minimizes unwanted changes in path length due to disturbances. A glass chamber is constructed to protect the interferometer from unwanted temperature drift and mechanical vibration. A chamber is designed to allow control for all necessary angular settings from the outside for all four subexperiments of the CHSH-inequality measurement, after which we performed a measurement in Alice and Bob's site with $|+\rangle /|-\rangle$ basis to confirm that we are still in the $\left|\Psi^{-}\right\rangle_{A B}$ state. Compactness of the setup, the Home-built chamber and temperature control $\left( \pm 1^{\circ}\right)$ enable keeping the phase stable for several minutes which is sufficient for our inequality measurement. Detection events are registered by a self-developed constant fraction discriminator (output signal width 2 ns) and self-developed counting module in combination with NIM-electronics for the logic.

In conclusion, our work supports that creation of entanglement between two independent photons is possible, even if there is no direct local interaction between the involved photons. The possible choices of the paths the photons take can be seen as the underlying cause for the observed nonlocal generation of entanglement. It is worth to remark that, together with synchronized independent narrow-band single photons [19] (typically have coherent length $5 \mathrm{~m}$ ) and active phase stabilization, our experiment can be extend to very long distance. The observed nonlocal two-photon interference and generation of entanglement are deeply rooted in the fundamentals of quantum mechanics and the question for hidden variables. Besides the interest to fundamental physics, the developed method has also practical applications, for example, non-locally generated entanglement can be directly utilized as essential resource for quantum-key distribution. If substituting independent photons for polarization entangled photons, we can readily prepare the two-photon four-dimensional entanglement and cluster state which can be employed to perform the test of 'all-versus nothing' quantum nonlocality [2022] and realize one-way quantum computation. [23, 24] Our results may find applications in all-optical quantum information processing.

[1] Einstein A, Podolsky B and Rosen N 1935 Phys. Rev. 47777

[2] Bell J 1964 Physics 1195

[3] Gisin N, Ribordy G, Tittel W and Zbinden H 2002 Rev. Mod. Phys. 74145

[4] Duan L M, Lukin M D, Cirac J I and Zoller P 2001 Nature 414 413

[5] Nielsen M A, Chuang I L 2002 Quantum Computation and Quantum Information (New York: Cambridge University)

[6] Kwiat P G et al 1995 Phys. Rev. Lett. 754337

[7] Freedman S J and Clauser J S 1972 Phys. Rev. Lett. 28938

[8] Rarity J G and Tapster P R 1990 Phys. Rev. Lett. 642495

[9] Shih Y H and Alley C O 1998 Phys. Rev. Lett. 612921

[10] Hong C K, Ou Z Y and Mandel L 1987 Phys. Rev. Lett. 592044

[11] Bennett C H et al 1993 Phys. Rev. Lett. 701895

[12] Zukowski M, Zeilinger A, Horne M A and Ekert A 1993 Phys. Rev. Lett. 714287

[13] Bose S, Vedral V and Knight P L, 1998 Phys. Rev. A 57, 822

[14] Pan J W, Bouwmeester D, Weinfurter H, Zeilinger A 1998 Phys. Rev. Lett. 803891

[15] Yurke B, Stoler D, 1992 Phys. Rev. A 462229

[16] Neder I, Ofek N, Chung Y, Heiblum M, Mahalu D and Umansky V 2007 Nature $\mathbf{4 4 8} 333$

[17] Boschi D, Branca S, Martini F De, and Hardy L 1997 Phys. Rev. Lett. 792755

[18] Clauser J, Horne M, Shimony S and Holt R 1969 Phys. Rev. Lett. 23880 
[19] Yuan Z S, Chen Y A, Chen S, Zhao B, Koch M, Strassel T, Zhao Y, Zhu G J, Schmiedmayer J and Pan J W 2007 Phys. Rev. Lett. 98180503

[20] Diao D S, Zhang Y S, Zhou X F, Guo G C 2008 Chin. Phys. Lett. 253555

[21] Chen Z B, Pan J W, Zhang Y D, Brukner C and Zeilinger A 2003 Phys. Rev. Lett. 90160408
[22] Yang T, Zhang Q, Zhang J, Yin J, Zhao Z, Zukowski M, Chen Z B andPan J W 2005 Phys. Rev. Lett. 95240406

[23] Walther P et al 2005 Nature (London) 434169

[24] Chen K, Li C M, Zhang Q, Chen Y A, Goebel A, Chen S, Mair A and Pan J W 2007 Phys. Rev. Lett. 99120503 